\title{
Semiclassical Treatment of a Half-Cycle Pulse Acting on a One- Dimensional Rydberg Atom
}

\author{
C. D. Schwieters
}

John B. Delos

William \& Mary, jbdelos@wm.edu

Follow this and additional works at: https://scholarworks.wm.edu/aspubs

Part of the Physics Commons

\section{Recommended Citation}

Schwieters, C. D. and Delos, John B., Semiclassical Treatment of a Half-Cycle Pulse Acting on a OneDimensional Rydberg Atom (1995). Physical Review A, 51(2), 1023-1029.

https://doi.org/10.1103/PhysRevA.51.1023

This Article is brought to you for free and open access by the Arts and Sciences at W\&M ScholarWorks. It has been accepted for inclusion in Arts \& Sciences Articles by an authorized administrator of W\&M ScholarWorks. For more information, please contact scholarworks@wm.edu. 


\title{
Semiclassical treatment of a half-cycle pulse acting on a one-dimensional Rydberg atom
}

\author{
C. D. Schwieters and J. B. Delos \\ Physics Department, The College of William and Mary, Williamsburg, Virginia 23187 \\ and Joint Institute for Laboratory Astrophysics, University of Colorado and National Institute of Standards and Technology, \\ Boulder, Colorado 80309
}

(Received 11 August 1994)

\begin{abstract}
The final-state distribution of hydrogen, acted upon by a $\frac{1}{2}$-cycle pulse, has been calculated semiclassically for a proposed one-dimensional experiment. This work was motivated by the recent experimental realization of half-cycle pulses by Jones, You, and Bucksbaum [Phys. Rev. Lett. 70, 1236 (1993)] in which preliminary studies of ionization and state redistribution for hydrogenlike atoms were carried out. To simplify the situation theoretically, an experiment is proposed in which an additional weak static electric field is imposed and approximately one-dimensional states are selected. Within this one-dimensional approximation the transition probability to various $n$ states ( $n$ is the principal quantum number) has been calculated as a function of the amplitude of the half-cycle pulse, using a semiclassical formula due to Miller [Adv. Chem. Phys. 25, 69 (1974)]. A complete derivation of this formula and a discussion of approximations are made in the following paper. We have found that an even number of trajectories contributes to the transition probability and leads to observable interference effects. In addition, we find that bifurcations of these trajectories can occur, resulting in more complicated interference structures.
\end{abstract}

PACS number(s): $03.65 . \mathrm{Sq}, 34.50 . \mathrm{Fa}, 32.80 . \mathrm{Rm}, 42.50 . \mathrm{Hz}$

\section{INTRODUCTION}

Many studies of the effects of ultra-high-intensity laser pulses on atoms have been carried out in recent years, and it is found that qualitatively new phenomena occur that are not predicted by perturbation theory when the radiation force is comparable to the binding Coulomb force [1]. Concomitant with the increase of the intensity of laser pulses has been a reduction in the pulse length. Recently, Jones et al. [2] have demonstrated a kind of limit of this pulse shortening by creating half-cycle pulses (HCP's) whose time profile looks approximately like the sine function with an argument from 0 to $\pi$.

Since the experimental demonstration of the HCP, several groups have performed quantum and classical simulations of the ionization probability and the probability of bound-bound transitions in Rydberg atoms under the action of such pulses [3-5]. These studies and preliminary experiments suggest a complex interference structure for the probability of transitions to energy-resolved final states.

In this paper we propose an experiment for which the atomic dynamics can be modeled as approximately one dimensional. For this model we calculate the probability of bound-bound transitions using a semiclassical method. The formulas give us a clear understanding of the rather complicated oscillatory behavior of the probability spectrum. Preliminary experiments have demonstrated the feasibility of the proposed experiment, but a detailed comparison with experiment is not yet possible.

In the current study, a static electric field is assumed to be present in addition to the HCP. The highest- and lowest-energy Stark states in this static field are elongated along the field axis and quite localized about this axis. Since the HCP gives a force that is directed along this same axis, we may neglect the transverse motion, and thereby reduce the problem to one-dimensional motion. This approximation has been used in the past to describe other experiments [6].

The goal of the current study is to calculate the probability of transition from one "blue" (high-energy) Stark state to another, or from one "red" (low-energy) Stark state to another, caused by a HCP.

Because the static-field case is integrable, it is particularly convenient to express the results in action and angle variables. The role of action and angle variables and general canonical transformations in quantum mechanics has a long history, but the theory is still not fully understood. However, in spite of the lack of rigorous basis in a fully quantum framework, action and angle variables are known to be useful for expressing the results of semiclassical approximations for separable and approximately separable systems. In the next section, we quote the result obtained by the method of Miller [7] when applied to the calculation of transition coefficients $a_{n}=\left\langle n \mid \psi\left(t_{f}\right)\right\rangle$. This formula yields the correct result, although it lacks a rigorous formulation. In Sec. III, we discuss the predictions of transition probabilities. We show that in most cases a simple oscillatory pattern arises because there are two classical orbits that go from each initial state to each final state. We also find an interesting situation in which two additional classical orbits from initial state to final state are created. This is a bifurcation of an orbit, and the simple semiclassical approximation fails at this point, 
so it must be replaced by an improved formulation. We give a rigorous justification for the formulas in a companion paper [8].

\section{THE TRANSITION PROBABILITY IN ACTION AND ANGLE VARIABLES}

The Hamiltonian for a one-dimensional hydrogen atom in parallel static and time-varying fields is

$$
H=\frac{1}{2 \mu} p^{2}-Z_{\mathrm{eff}} \frac{e^{2}}{z}+e z\left[\mathcal{F}_{1}+\mathcal{F}_{2}(t)\right]
$$

Equation (1) is derived and its range of validity is discussed in the companion paper. The single active electron of mass $\mu$ is assumed to be moving on the positive $z$ axis, where it experiences a nuclear charge of $Z_{\text {eff }}$, a weak static field $\mathcal{F}_{1}$, and the HCP $\mathcal{F}_{2}(t)$

$$
\mathcal{F}_{2}(t)= \begin{cases}\mathcal{F}_{2} \sin \left(\omega_{F} t\right), & 0<t \leq t_{p}=\frac{\pi}{\omega_{F}} \\ 0, & t<0, t>t_{p}\end{cases}
$$

In the current study, the length of the HCP, $t_{p}$, was taken as $1 / 2$ the orbital period of the initial (unperturbed) system.

A positive value of $\mathcal{F}_{1}$ corresponds to the blue (highenergy) state, while a negative value of $\mathcal{F}_{1}$ corresponds to a red (low-energy) state with a lowered barrier to ionization. For either case, $\mathcal{F}_{2}$ can have either sign: $\mathcal{F}_{2}>0$ means that the electric force of the HCP is towards the nucleus, while $\mathcal{F}_{2}<0$ means that it is away. In any case, the electron is confined to the positive $z$ axis: when it hits the Coulomb singularity it goes back in the direction from which it came. In principle, the behavior of the electron depends upon both the sign of $\mathcal{F}_{1}$ and the sign of $\mathcal{F}_{2}$. However, in our calculations, we take $\mathcal{F}_{1}$ sufficiently small that it does not affect the orbit during the pulse: its only role is to define the initial and final states as red or blue (linear) Stark states.

Equation (1) contains a hitherto unrecognized correction [8]. In previous applications of this one-dimensional approximation, the nuclear charge $Z_{\text {eff }}$ was taken to be unity. Our analysis in the companion paper shows that zero-point motion in the (otherwise ignored) degree of freedom transverse to the $z$ axis leads to a small correction, and we should take $Z_{\text {eff }}=Z-\beta / 2 e^{2}$, where $\beta$ is a separation constant.

In the semiclassical approximation, we compute trajectories of the electron under this Hamiltonian, and then connect those trajectories to quantum states. For this purpose we define action and angle variables using the time-independent part of the Hamiltonian (i.e., $\mathcal{F}_{2}=0$ )

$$
\begin{gathered}
I=\frac{1}{2 \pi} \oint p_{z}(z ; E) d z \\
\Theta=\mu \omega(E) \int \frac{1}{p_{z}(z ; E)} d z-\omega(E) t
\end{gathered}
$$

where

$$
\begin{gathered}
p_{z}(z ; E)=\left\{2 \mu\left[E-V_{0}(z)\right]\right\}^{1 / 2} \\
V_{0}(z)=-Z_{\text {eff }} e^{2} / z+e z \mathcal{F}_{1}
\end{gathered}
$$

and $\omega(E)$ is $2 \pi$ divided by the orbital period. Semiclassical quantization picks out $I=(n-1 / 2) \hbar$, where $n$ is a positive integer, as allowed values of action, i.e., the red or blue Stark state with principal quantum number $n$ corresponds to a periodic orbit on the $z$ axis having $(n-1 / 2) \hbar$ units of action.

The set of all points on this periodic orbit is represented by a line of constant action $I=I_{0}$ having any phase angle between zero and $2 \pi$ [solid curve in Fig. 1(b)]. This line is called the "initial Lagrangian manifold."

The effect of a time-dependent electric field is to deform this line into a smooth curve, the "final Lagrangian manifold." Examples of time-evolved manifolds are shown as the dotted and dashed lines in Fig. 1(b). This curve does not evolve after the time-dependent field has been turned off.

It is best to plot $I_{f}$, the final action after the pulse, versus the initial angle at the time the pulse is turned on, $\Theta_{0}$, and this is shown as the dotted and dashed lines in Fig. 1(a). This curve also remains constant after the external perturbation has been turned off; it represents at any time after the pulse the final action of the trajectories whose initial phase was $\Theta_{0}$. Obviously, $I_{f}\left(\Theta_{0}\right)$ must be a smooth function, and it must be periodic in $\Theta_{0}$ with period $2 \pi$.

We wish to calculate the probability amplitude for transition from a Stark state having principal quantum number $n_{0}$ to one having principal quantum number $n_{f}$. To do this we identify those values of $\Theta_{0}$ (called $\Theta_{k}$ ) such that $I_{f}\left(\Theta_{0}\right)=\left(n_{f}-1 / 2\right) \hbar$. In Figs. 1(a) and 1(b), these values of $\Theta_{0}$ can be read from the intersections of the dashed and dotted lines with the desired value of final action. Then the semiclassical formula for this transition coefficient can be written as [7]

$$
\begin{aligned}
a_{n}= & \sqrt{\frac{\hbar}{2 \pi}} \sum_{\Theta_{k}}\left(-i \frac{\partial I_{f}\left(\Theta_{0}\right)}{\partial \Theta_{0}}\right)_{\Theta_{0}=\Theta_{k}}^{-1 / 2} \\
& \times \exp \left\{\frac{i}{\hbar} \int_{0}^{t_{f}}\left[-\Theta(t) \frac{d I(t)}{d t}-E(t)\right] d t\right\} .
\end{aligned}
$$

The sum is over the classical paths that start at $\left(I=I_{0}\right.$; $\left.\Theta_{0}=\Theta_{k}\right)$ and end with $I\left(t_{f}\right)=I_{f}$. The argument of the exponential is a classical action integrated along each such path. The square root in the preexponential factor is defined to have the phase convention

$$
(-i a)^{-1 / 2}=|a|^{-1 / 2} e^{+i(\pi / 4) \operatorname{sgn} a} .
$$

The amplitude of each contribution to the transition coefficient is $\sqrt{\hbar /(2 \pi)}\left|\partial I / \partial \Theta_{0}\right|^{-1 / 2}$. Hence, when the slope of the curve of $I_{f}$ versus $\Theta_{0}$ is large, the transition amplitude is small, while at the extrema where the slopes go to zero, this primitive semiclassical approximation breaks down and the amplitude goes to infinity.

There are two contributions to the phase of the tran- 
sition coefficient: one due to a classical actior integrated along the trajectory in action and angle variables and the other an additional "Maslov" contribution from Eq. (7). Since the transition probability is the square modulus of
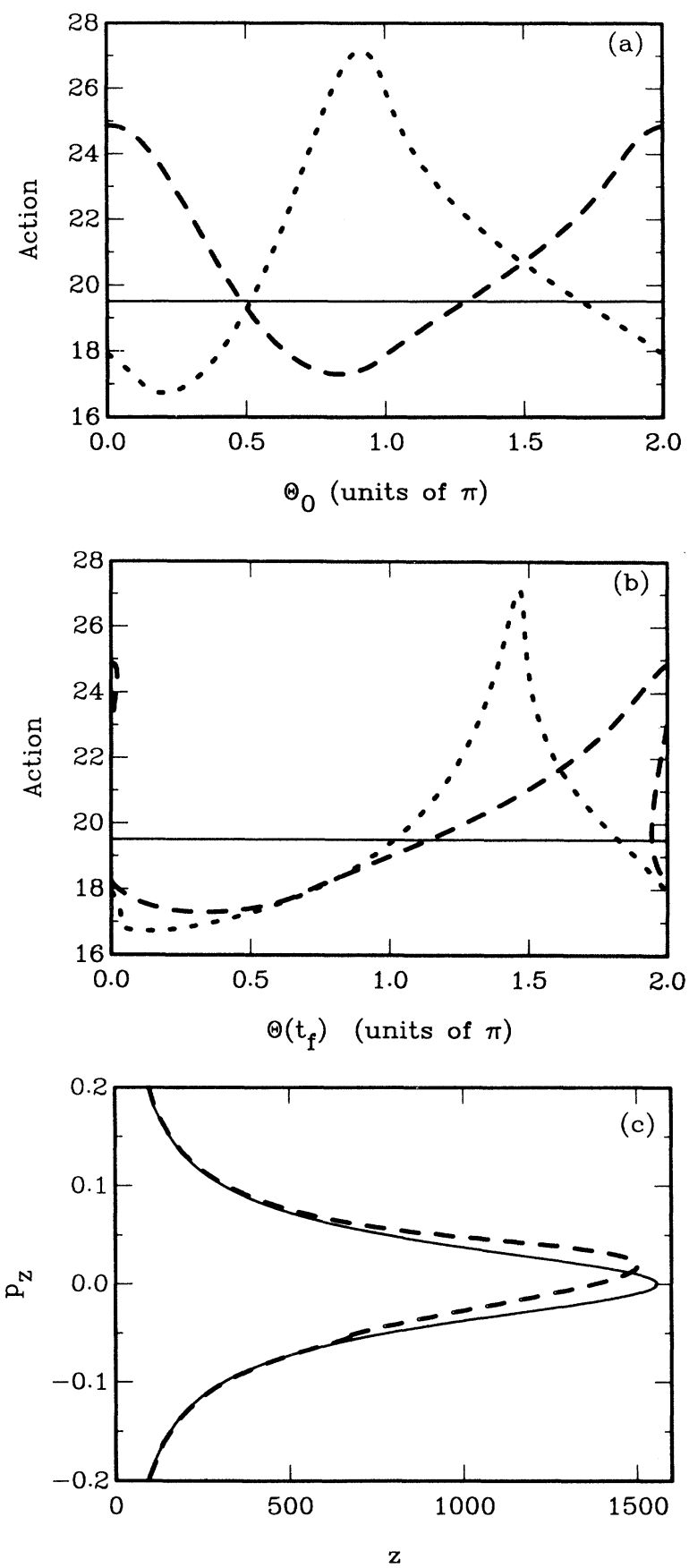

FIG. 1. Manifolds for $I_{0}=19.5,\left|\mathcal{F}_{2}\right|=1 \times 10^{-6}$ a.u. (a) These curves represent $I\left(t_{f}\right)$ (the final action) versus $\Theta_{0}$, the initial angle. The thin straight line curve is the manifold of initial conditions, while the evolved manifold for $\mathcal{F}_{2}>0$ $\left(\mathcal{F}_{2}<0\right)$ is shown by the dashed (dotted) curve. (b) Here $I\left(t_{f}\right)$ is plotted versus $\Theta\left(t_{f}\right)$, the angle at the end of the pulse. (c) The initial (solid) and evolved (dashed) manifolds in $\left(p_{z}, z\right)$ coordinates for $\mathcal{F}_{2}>0$. the transition coefficient, phase differences between different trajectories which contribute to a given transition probability are the quantities which have physical significance. The integral quantity in Eq. (7) is independent of path, so the difference between two contributions for $I_{f}=I_{n}$ corresponds to areas traced out between the final time manifold [the dotted and dashed lines in Fig. 1 (b) ] and the straight line $I=I_{n}=\hbar(n-1 / 2)$. The sign of the slope of $I\left(t_{f}\right)$ versus $\Theta_{0}$ alternates at consecutive intersections so that the Maslov part of the phase difference is always $e^{ \pm i \pi / 2}$. We give a complete semiclassical derivation of Eq. (7) in the companion paper.

\section{RESULTS}

In this section we show and discuss some results for hydrogen acted upon by a HCP using the procedure outlined in the previous section.

\section{A. Interference effects in excitation}

Figure 1 contains plots of action versus angle for the initial manifold as well as plots of the manifold after interacting with two different HCP's. In this case the initial state corresponds to $n=20, \mathcal{F}_{1}$ small, $\left|\mathcal{F}_{2}\right|=1 \times 10^{-6}$ a.u., and the pulse length is half of the $(n=20)$ Kepler period. As mentioned before, the initial manifold for $n=20$ is a horizontal line at $I=19.5$ which contains the initial conditions for all the trajectories. When the HCP is applied to the system, the manifold is distorted. Plots of $I_{f}$ versus $\Theta_{0}$ are shown for both $\mathcal{F}_{2}>0$ (dashed curve) and $\mathcal{F}_{2}<0$ (dotted curve)in Fig. 1(a), while $I_{f}\left(\Theta_{f}\right)$ is plotted in Fig. 1(b). The corresponding initial manifold and time-evolved manifold for $\mathcal{F}_{2}>0$ are plotted in Fig. 1 (c) for comparison.

Since the final curve $I_{f}\left(\Theta_{0}\right)$ must represent a periodic function, in general there will be an even number of orbits from the initial state to any given final state. In Fig. 1(a) both final time curves intersect any given value of action either zero or two times. There are isolated points with one intersection and zero slope at the maxima or minima of $I_{f}\left(\Theta_{0}\right)$, and the primitive semiclassical approximation fails at those points.

The spectrum of transition probabilities corresponding to the two final time curves in Figs. 1(a) and 1(b) are shown in Fig. 2 along with their purely classical counterparts. Equation (7) can be calculated for a continuous range of values of $n$ (not just integers), and this is also shown in Fig. 2. One can see that interference between the two trajectories leads to oscillations in the transition probabilities. For this pulse, only states with small $\Delta n$ are populated, but the initial state does not retain the largest population after the pulse for either sign of $\mathcal{F}_{2}$. Note that Eq. (7) will not predict the transition probabilities due to tunneling: there is an abrupt discontinuous change to zero transition probability for values of action larger or smaller than those spanned by the manifold $I_{f}\left(\Theta_{0}\right)$.

In Fig. 3, we examine one peak in the transition prob- 


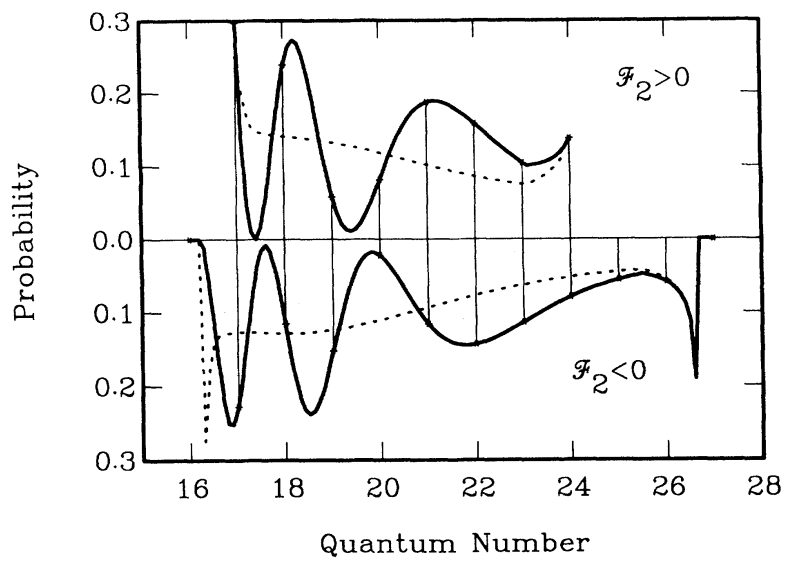

FIG. 2. Spectrum of transition probabilities for $I_{u}=19.5$, $\left|\mathcal{F}_{2}\right|=1 \times 10^{-6}$. Semiclassical probabilities are plotted as sticks and the purely classical probability is shown as dotted curves. Results for $\mathcal{F}_{2}>0$ are shown above the axis while those for $\mathcal{F}_{2}<0$ are shown below the axis. The solid curves represent semiclassical transition probabilities at nonquantized values of action.

ability spectrum and vary the magnitude of $\mathcal{F}_{2}$. Specifically, we show the survival probability in the initial state $n=20$ for $\mathcal{F}_{2}<0$ (force away from the nucleus). The fields considered here are very strong: for comparison, the static field that would fully ionize the $n=20$ state over the barrier is about $-4 \times 10^{-7}$ a.u. Accordingly, the survival probability in the initial state is small: around $5 \%$. Of course, for small $\mathcal{F}_{2}$ the survival probability gets large (it should go to 1), and the semiclassical formula [Eq. (7)] breaks down. The oscillations again arise from the fact that precisely two initial angles lead to $I_{f}=I_{0}$.

Figure 4 shows the survival probability for $n_{0}=80$ for both signs of the HCP, whose duration is $1 / 2$ the $n=80$ orbital period. It is seen that these two spectra are similar over a large range of $\mathcal{F}_{2}$. In this case the static

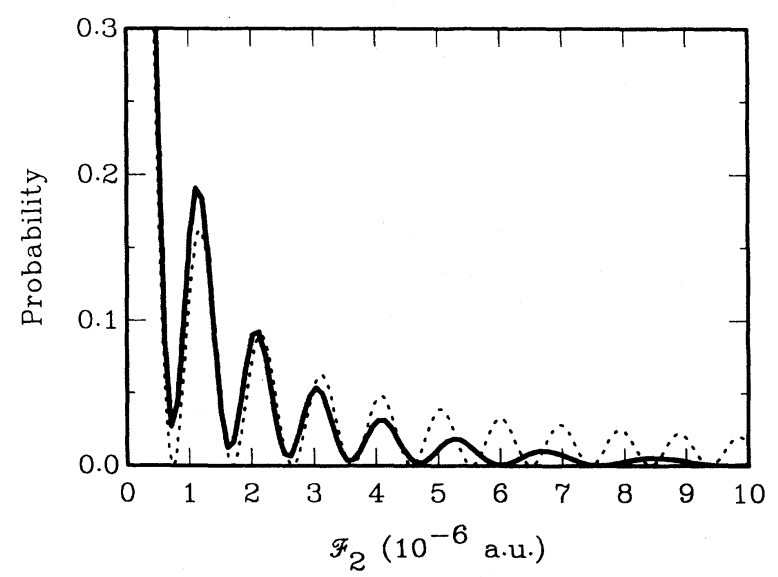

FIG. 3. The survival probability of the $n_{0}=20 \rightarrow n_{f}=20$ transition. Plotted is the spectrum for $\mathcal{F}_{2}<0$ (solid curve) and the result due to Jensen [5] using the strong correspondence principle (dotted curve).

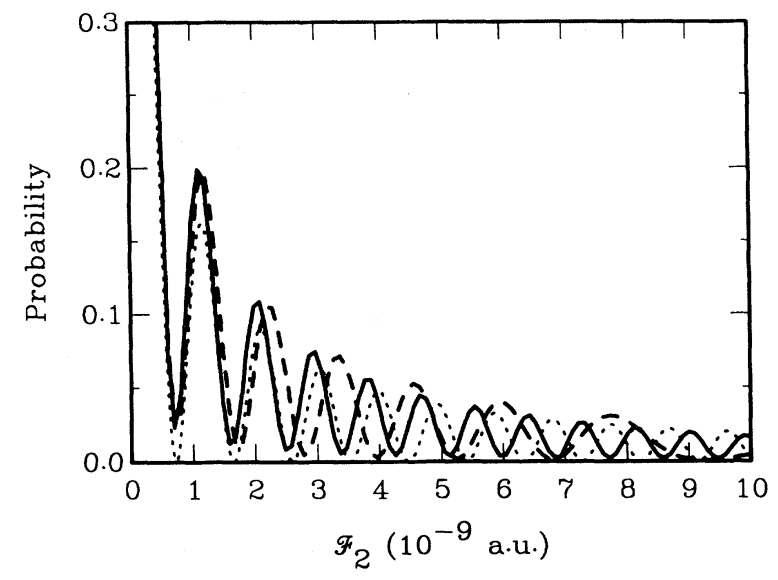

FIG. 4. Same as Fig. 3, except that the initial and final states correspond to $n=80$. In addition, the spectrum for $\mathcal{F}_{2}>0$ is plotted as the dashed curve.

electric field strength for ionization is $-1.5 \times 10^{-9}$.

Jensen [5] has applied the strong correspondence principle discussed by Percival and Richards [9] to this problem. It gives an analytic expression for the transition probability and for the survival probability in terms of Bessel functions. The formula is more accurate than Eq. (7) for small $\mathcal{F}_{2}$ (it correctly gives $P_{\text {survival }}=1$ when $\mathcal{F}_{2} \rightarrow 0$ ), but it is less accurate than Eq. (7) for large $\mathcal{F}_{2}$ or large changes in $n$ because it is based on an assumption that the orbit is only slightly disturbed by the pulse. The formula predicts that the survival and transition probabilities are independent of the sign of $\mathcal{F}_{2}$ and depend only on the magnitude of $|\Delta n|$ : the transition probability for 20 to 16 should be equal to that from 20 to 24. Our calculations show that both of these predictions are reasonably accurate when $\mathcal{F}_{2}$ and $|\Delta n|$ are not too large. Jensen's result is plotted in Figs. 3 and 4 as the dotted curve and shows reasonably good agreement with our results in the plotted range.

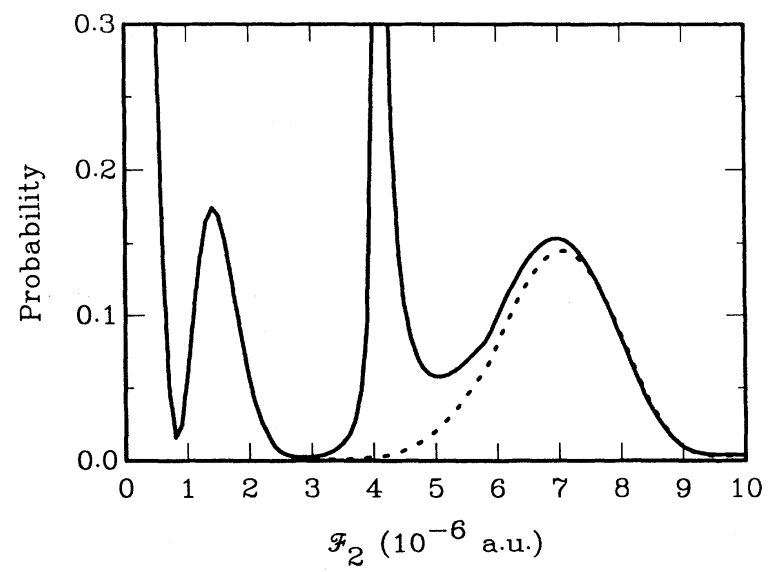

FIG. 5. The uniform (dotted curve) and primitive (solid curve) semiclassical $n=20 \rightarrow n=20$ survival probability for $\mathcal{F}_{2}>0$. 


\section{B. A Bifurcation}

We discovered an interesting phenomenon when we considered the case $\mathcal{F}_{2}>0$ (force toward the nucleus). Figure 5 shows the survival probability for $n_{0}=n_{f}=20$ for this case. For small $\mathcal{F}_{2}$ the survival probability is similar to that found in Fig. 3 , but around $\mathcal{F}_{2}=3 \times 10^{-6}$ a.u. the survival probability becomes very small; then it rises again. The primitive semiclassical approximation (solid curve) gives a singularity near $\mathcal{F}_{2} \approx 4.1 \times 10^{-6}$ a.u., and then it rises to about 0.15 near $\mathcal{F}_{2}=7 \times 10^{-6}$ a.u. A uniform semiclassical approximation, discussed in the next section, eliminates the singularity, but leaves the peak at $\mathcal{F}_{2}=7 \times 10^{-6}$ a.u. ${ }^{1}$

This phenomenon is investigated further in Fig. 6. We see that, as $\mathcal{F}_{2}$ increases, the curve $I_{f}\left(\Theta_{0}\right)$ develops a local cubic structure near $\Theta_{0}=0.9 \pi$ : for small $\mathcal{F}_{2}$ the curve has two intersection points with the line $I_{f}=19.5$, for large $\mathcal{F}_{2}$ it has four such intersections, and at a critical value of $\mathcal{F}_{2}$, the curve has an inflection point with zero slope at $I_{f}=19.5$. At this critical field strength, two new classical orbits that begin and end at $I=19.5$ are created. We call it, therefore, a bifurcation of an open orbit from the initial to the final action.

A peculiar feature of the bifurcation illustrated in Fig. 6 is that the two new trajectories are created exactly at $I_{0}=I_{f}=19.5$. This is "strange" because, even if we find the kind of bifurcation indicated by the graphs in Fig. 6, there is no reason to think that the zero-slope point will exactly coincide with the initial state. This coincidence indicates that we need to look deeper.

To understand this phenomenon, we need to examine

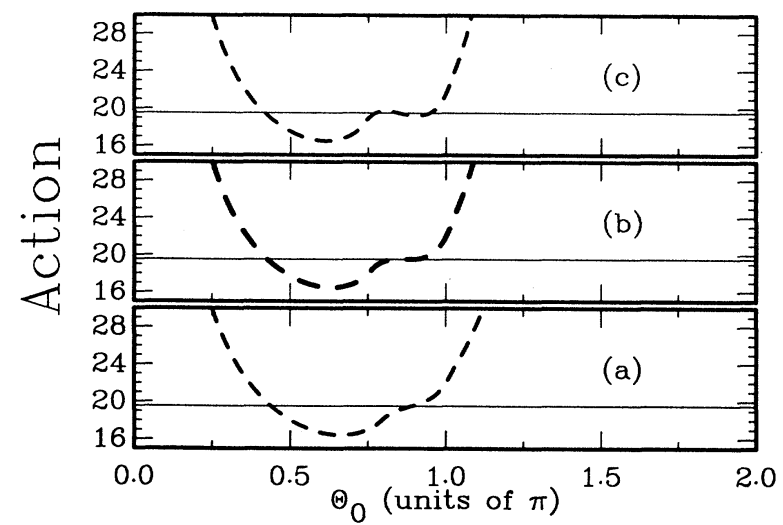

FIG. 6. Plots of $I_{f}$ versus $\Theta_{0}$ for $\mathcal{F}_{2}>0$ : (a) $\mathcal{F}_{2}=3.5 \times 10^{-6}$ (below bifurcation), (b) $\mathcal{F}_{2}=4.1 \times 10^{-6}$ (at bifurcation), and (c) $\mathcal{F}_{2}=4.5 \times 10^{-6}$ (above bifurcation).

\footnotetext{
${ }^{1}$ Arguments given in the Appendix of the companion paper consider the question of whether the one-dimensional (1D) approximation is reliable in this case. We give arguments which show that this approximation should be valid for $\mathcal{F}_{\mathbf{2}} \lesssim$ $2.6 \times 10^{-6}$ a.u. and we present $2 \mathrm{D}$ calculations which show that it is at least qualitatively correct for stronger fields.
}

the orbits in real space. In Fig. 7 we show the unperturbed linear orbit as a loop. For very small (positive) $\mathcal{F}_{2}$ the the trajectories are not significantly perturbed away from the zero-HCP loop. Contributions to the survival probability spectrum consist of those orbits which begin and end with the same energy: all energy taken away from the electron by the HCP must be returned, or vice versa. Thus, initially there are only two trajectories which contribute to the survival probability, as shown in Fig. 7(a). Due to the time-symmetric nature of the HCP, the orbits are at turning points at the middle point of the pulse, $t=t_{1 / 2}$, and are symmetric in time and space about these points:

$$
\begin{aligned}
z\left(t_{1 / 2}-t\right) & =z\left(t_{1 / 2}+t\right), \\
p_{z}\left(t_{1 / 2}-t\right) & =-p_{z}\left(t_{1 / 2}+t\right) .
\end{aligned}
$$

The trajectory which is at the nucleus at $t=t_{1 / 2}$ we call $z_{1}\left(t ; \mathcal{F}_{2}\right)$ while that which is at the outer turning point we call $z_{2}\left(t ; \mathcal{F}_{2}\right)$.

Consider $z_{2}$, the dashed trajectory in Fig. 7. At the instant the pulse begins, the electron is at the marked point, and it is moving upward, away from the nucleus. As the pulse turns on, the electron is decelerated until it reaches a turning point somewhat below the unperturbed loop, and then it is accelerated as it comes back downwards. The speed and phase of the electron and the shape and strength of the pulse are precisely such that the electron reaches the outer turning point of its orbit exactly when the pulse reaches a maximum.

What happens if the initial phase of the electron is changed? A small increase in $\Theta_{0}$ will cause the trajectories to spend less time on the right (energy-losing) side and more on the left side, where energy is gained, so that $d I_{f} / d \Theta_{0}$ is positive. This is consistent with Fig. 6(a). Now consider what happens if we increase $\mathcal{F}_{2}$ by a small amount $\delta \mathcal{F}_{2}$. The trajectory whose initial position on the manifold is at $z=z_{2}\left(t=0 ; \mathcal{F}_{2}\right)$ will initially be decelerated more than $z_{2}\left(t ; \mathcal{F}_{2}\right)$ and will thus reach the outer turning point before $t_{1 / 2}$. Therefore, we must have $z_{2}\left(t=0 ; \mathcal{F}_{2}+\delta \mathcal{F}_{2}\right)<z_{2}\left(t=0 ; \mathcal{F}_{2}\right)$. Eventually, for large enough $\mathcal{F}_{2}, z_{2}\left(t=0 ; \mathcal{F}_{2}\right)$ will reach the nucleus. Then matters become more complicated [Fig. 7(d)]. For larger values of $\mathcal{F}_{2}$ the electron has its energy initially increased (while on the left side) and then decreased after it passes the nucleus and before it reaches $t=t_{1 / 2}$. At some still larger value of $\mathcal{F}_{2}$, the energy lost by a trajectory starting at $\Theta_{0}+\Delta \Theta_{0}$ spending less time on the right side of the nucleus will be larger than that gained on the left side, and the slope of $d I_{f} / d \Theta_{0}$ will pass through zero. The value of $\mathcal{F}_{2}$ at which this occurs depends on the exact shape of the HCP. For values of $\mathcal{F}_{2}$ larger than this critical value $z_{2}$ spawns two daughter trajectories, $z_{2,1}$ and $z_{2,2}$, neither of which retain the symmetry about $t=t_{1 / 2}$ of the HCP. Instead, these trajectories are related to each other by the symmetry $z_{2,1}\left(t_{1 / 2}-t\right)=z_{2,2}\left(t_{1 / 2}+t\right)$.

We have shown that the symmetry about $t_{1 / 2}$ of our form for the HCP is responsible for the bifurcation occurring at $I_{f}=I_{0}$. Laboratory realizations of HCP's do not possess this symmetry and therefore the bifurcation will occur at some value of action somewhat different than $I_{0}$ in experiments. 


\section{Schematic of Trajectories}

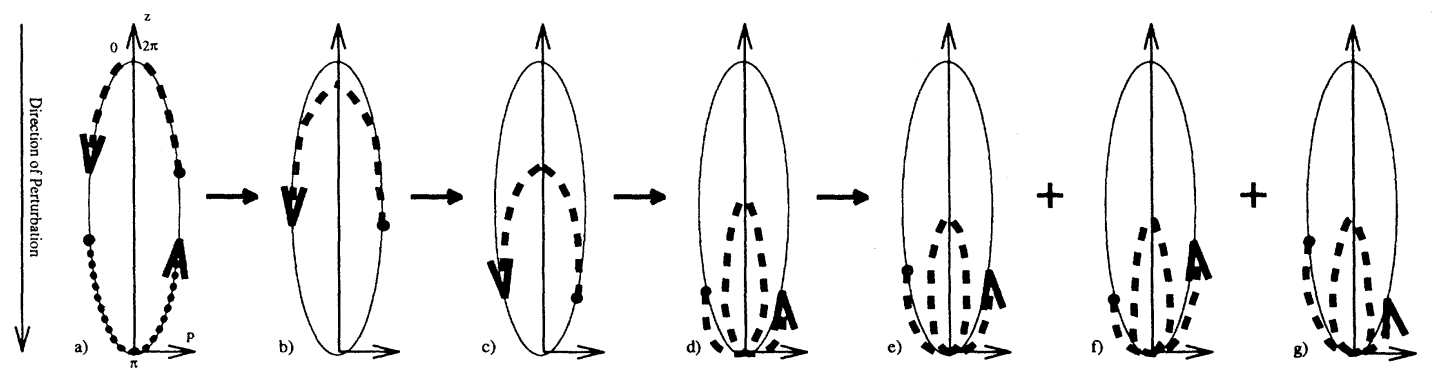

FIG. 7. Schematic sketches of trajectories for different values of $\mathcal{F}_{2}>0$. The thin loop is the unperturbed trajectory, while the dashed and dotted curves denote the two trajectories which contribute to the survival probability spectrum below bifurcation. The numbers on panel (a) indicate the conventions used for $\Theta_{0}$. Larger $\Theta_{0}$ corresponds to smaller $z(t=0)$ for $p<0$ and larger $z(t=0)$ for $p>0$. The strength of the perturbation is successively larger in each panel until the bifurcation occurs and two daughters [shown in panels (f) and $(\mathrm{g})]$ are born. Note that this is just a schematic: in real phase space $p_{z}$ is discontinuous at the nucleus.

\section{UNIFORM APPROXIMATION}

In the region near the bifurcation, the primitive semiclassical approximation used in the previous section breaks down, as reflected in the singularity of the solid curve in Fig. 5. The quantum formula for the transition coefficient is an integral, $a_{n}=\left\langle n \mid \psi\left(t_{f}\right)\right\rangle$, and in the companion paper we show that this integral is approximately equal to

$$
a_{n}=\int \hat{f}\left(\Theta_{0}\right) \exp \left[\frac{i}{\hbar} \hat{g}\left(\Theta_{0}\right)-i \hat{\mu} \frac{\pi}{2}\right] d \Theta_{0}
$$

where $\hat{f}\left(\Theta_{0}\right)$ and $\hat{g}\left(\Theta_{0}\right)$ are functions that can be obtained from the final Lagrangian manifolds, $I_{f}\left(\Theta_{0}\right)$ and $\Theta_{f}\left(\Theta_{0}\right)$. If Eq. (11) is evaluated using the stationaryphase approximation, the primitive semiclassical approximation [Eq. (7)] is obtained. At the points of bifurcation, a higher-order approximation is needed. Connor [10], following work of others [11], has introduced a systematic method of applying uniform methods to the evaluation of integrals of the form in Eq. (11).

Results of this uniform calculation are shown in Fig. 5 as the dotted curve. It is seen that the singularity in the primitive semiclassical survival probability is replaced by a smooth curve of low probability in the uniform calculation. One usually expects classical singularities to be replaced by a peak in the quantum probability of finite amplitude; for example, in a harmonic oscillator, there is a large but finite quantum probability near a classical turning point. However, the quantum maximum is displaced somewhat from the classical singularity. In the present case, the quantum probability has a maximum amplitude at $\mathcal{F}_{2} \approx 7 \times 10^{-6}$, while the bifurcation occurs at $\mathcal{F}_{2} \approx 4.1 \times 10^{-6}$. One would expect that for smaller values of $\hbar$ (which under classical scaling occur for larger values of $n$ ) the resonance would move closer to the classical singularity. (One must also remember that the transition coefficient is a sum of interfering terms from the bifurcating trajectory and a nonbifurcating trajectory, and this also shifts the maximum.)

In the bifurcation treated here and illustrated in Fig. 5, two new trajectories were created out of one. For transitions for which $\Delta n \neq 0$, other bifurcations occur whereby two orbits are created from none. These types of bifurcations can also be treated using uniform semiclassical methods. In general, there is a sequence of bifurcations as $\mathcal{F}_{2}$ is increased, only one of which is illustrated in the current study.

\section{CONCLUSION}

In this paper we have proposed an experiment in which one-dimensional hydrogen in some initial pure state is subjected to a HCP and the transition probability to different final states is measured. We calculate the expected results using the semiclassical formula of Miller. Because there is always an even number of classical paths leading from an initial quantum state to a final state, we conclude that interference between these trajectories leads to an oscillatory spectrum. In addition, we found that the contributing trajectories can bifurcate, resulting in a discernible signature in the spectrum. Finally, while we treated only the red and blue Stark states of hydrogen in this study, this method can be extended in a straightforward manner to deal with arbitrary states of hydrogen.

\section{ACKNOWLEDGMENTS}

This work was supported by the Office of Naval Research and the National Science Foundation. We thank JILA for their hospitality. 
[1] For a recent review, see K. Burnett, V. C. Reed, and P. L. Knight, J. Phys. B 26, 561 (1993).

[2] R. R. Jones, D. You, and P. H. Bucksbaum, Phys. Rev. Lett. 70, 1236 (1993); and unpublished.

[3] C. O. Reinhold, M. Melles, H. Shao, and J. Burgdörfer, J. Phys. B 26, L1 (1993).

[4] K. J. LaGattuta and P. B. Lerner, Phys. Rev. A 49, R1547 (1994).

[5] R. Jensen (private communication).

[6] J. E. Bayfield and L. A. Pinnaduwage, Phys. Rev. Lett.
54, 313 (1985).

[7] W. H. Miller, Adv. Chem. Phys. 25, 69 (1974).

[8] C. D. Schwieters and J. B. Delos, following paper, Phys. Rev. A 51, 1030 (1995)].

[9] I. C. Percival and D. Richards, J. Phys. B 3, 1035 (1970).

[10] J. N. L. Connor, Mol. Phys. 26, 1217 (1973).

[11] C. Chester, B. Friedman, and F. Ursell, Proc. Cambridge Philos. Soc. Math. Phys. Sci. 53, 599 (1957); F. Ursell, Proc. Cambridge Philos. Soc. 72, 49 (1972). 